\title{
Electronic and Magnetic Properties of Kagome Metal $\mathrm{MgFe}_{6} \mathrm{Ge}_{4}$
}

Alisha Budhathoki 1,2, Madhav Prasad Ghimire ${ }^{2,3, *}$

${ }^{1}$ Department of Physics, University of Illinois at Chicago, Illinois 60607, U.S.A.

${ }^{2}$ Central Department of Physics, Tribhuvan University, Kirtipur 44613, Kathmandu, Nepal. ${ }^{3}$ IFW Dresden, Helmholtzstr-20, 01069 Dresden, Germany.

*madhav.ghimire@cdp.tu.edu.np
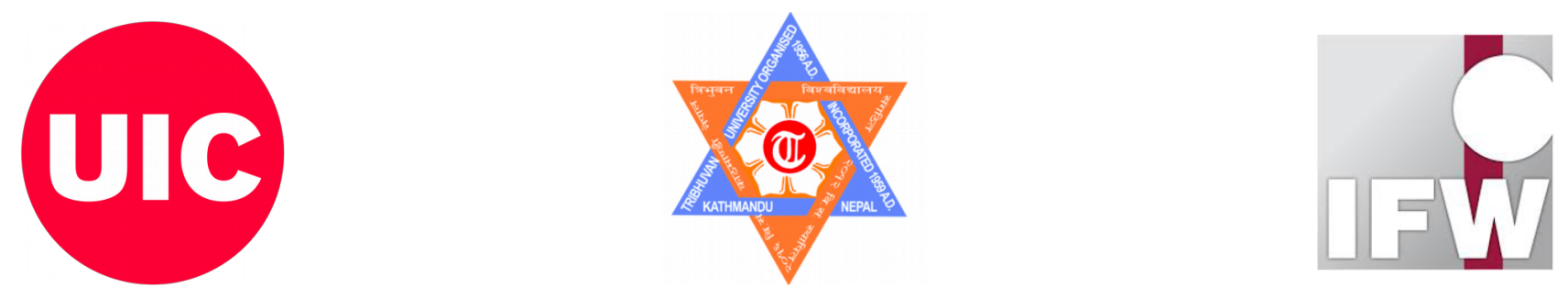


\section{Outline}

- Abstract

- Introduction

- Computational Methods

- Results and Discussions

- Conclusions

- References 


\section{Abstract}

Wide range of investigation is going on to study kagome lattice to create device with perfect conductivity. Here, we perform density functional calculation using FPLO code to study electronic and magnetic properties of $\mathrm{MgFe}_{6} \mathrm{Ge}_{4}, \mathbf{a}$ kagome system, where we noticed the material to be ferromagnetic with a total magnetic moment of $11.38 \mu_{\mathrm{B}} / \mathrm{unit}$ cell. We found the metallic nature where Fe3d shows the highest contribution at Fermi level in total DOS. Hybridization between Fe-3d and Ge-4p is observed around Fermi level. The Wannier fitting with the DFT calculations was performed to obtain wannier Hamiltonian to explore the Weyl points in $\mathrm{MgFe}_{6} \mathrm{Ge}_{4}$.

Keywords: Kagome lattice; Density functional theory 


\section{Introduction}

\section{Kagome Metal}

$\rightarrow \quad$ Word kagome derived from japanese kagome basket

$\rightarrow \quad$ Two dimensional network of corner-sharing triangle

$\rightarrow$ Exhibit novel properties like anamolous hall effect, quantum anamolous hall effect

$\rightarrow$ Possibility of metals with kagome lattice in quantum computing

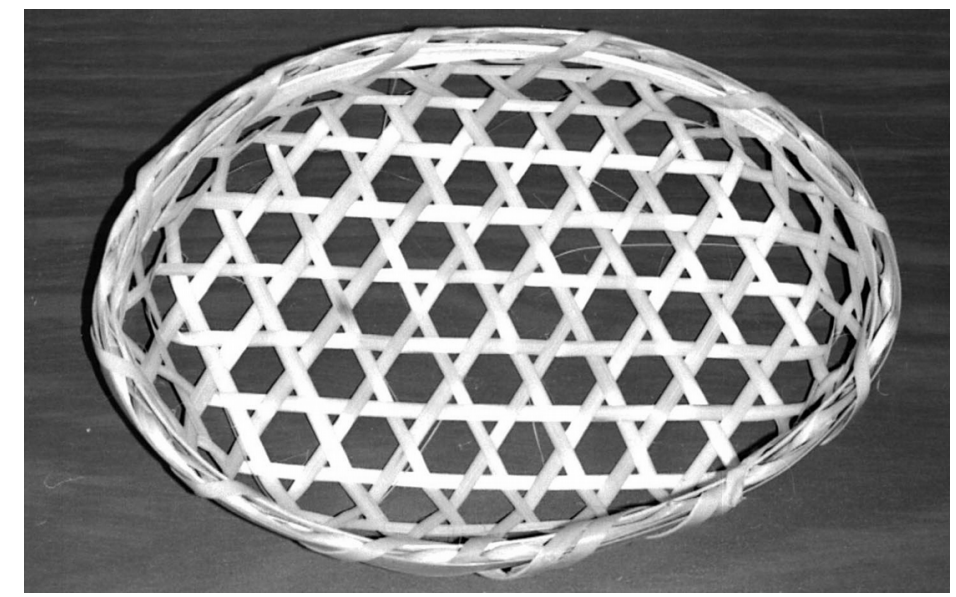

Fig 1: Bamboo basket woven in kagome pattern Physics today (2003) 


\section{Computational Methods}

- Density Functional theory calculation is performed in the fullpotential local orbital code (FPLO).

K. Koepernik and H. Eschrig , Phy. Rev. B 59, 1743 (1999)

- Scalar-relativistic and Full relativistic calculation was performed for 6 * 6 * $6 \mathrm{k}$-mesh.

- Standard generalized gradient approximation (GGA) is considered.

- Tight binding Hamiltonian is obtained by considering Wannier band mapping with FPLO. 
Results and Discussion

Crystal Information

- Hexagonal Crystal

- Experimental lattice parameter

- $a=b=5.09 \AA, c=20.10 \AA$

Matar et al., Solid State Sciences, (2015)

- $\alpha=\beta=90^{\circ}, y=120^{\circ}$

- Space group: $\mathbf{R} \overline{3} m(166)$

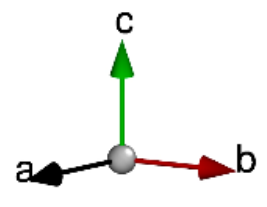

(Mg)

Mg

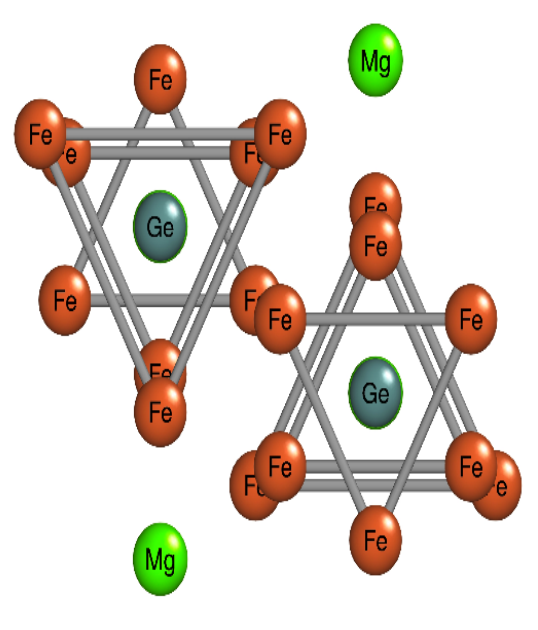

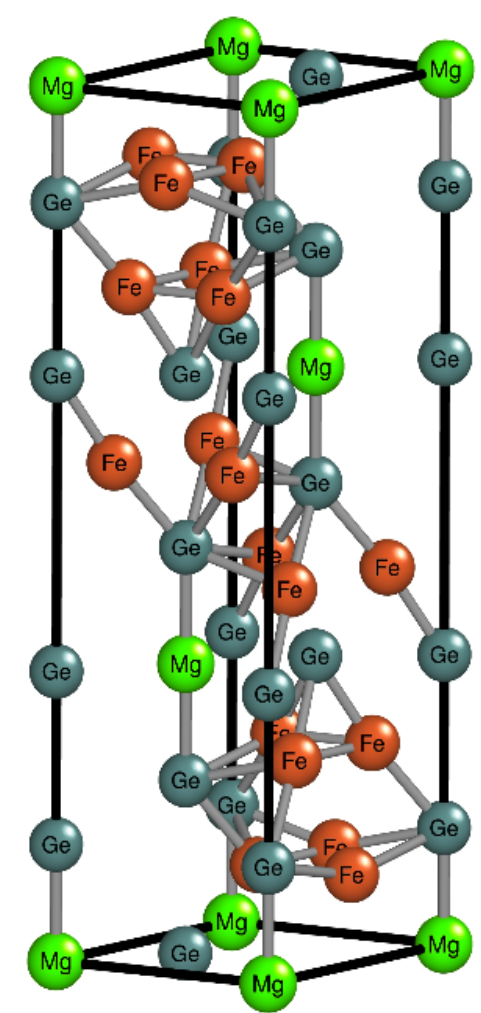

Fig 3: Crystal Structure of $\mathrm{MgFe}_{6} \mathrm{Ge}_{4}$

Fig 4: Kagome structure formed by Network of Fe atoms in $\mathrm{MgFe}_{6} \mathrm{Ge}_{4}$ 
(a)

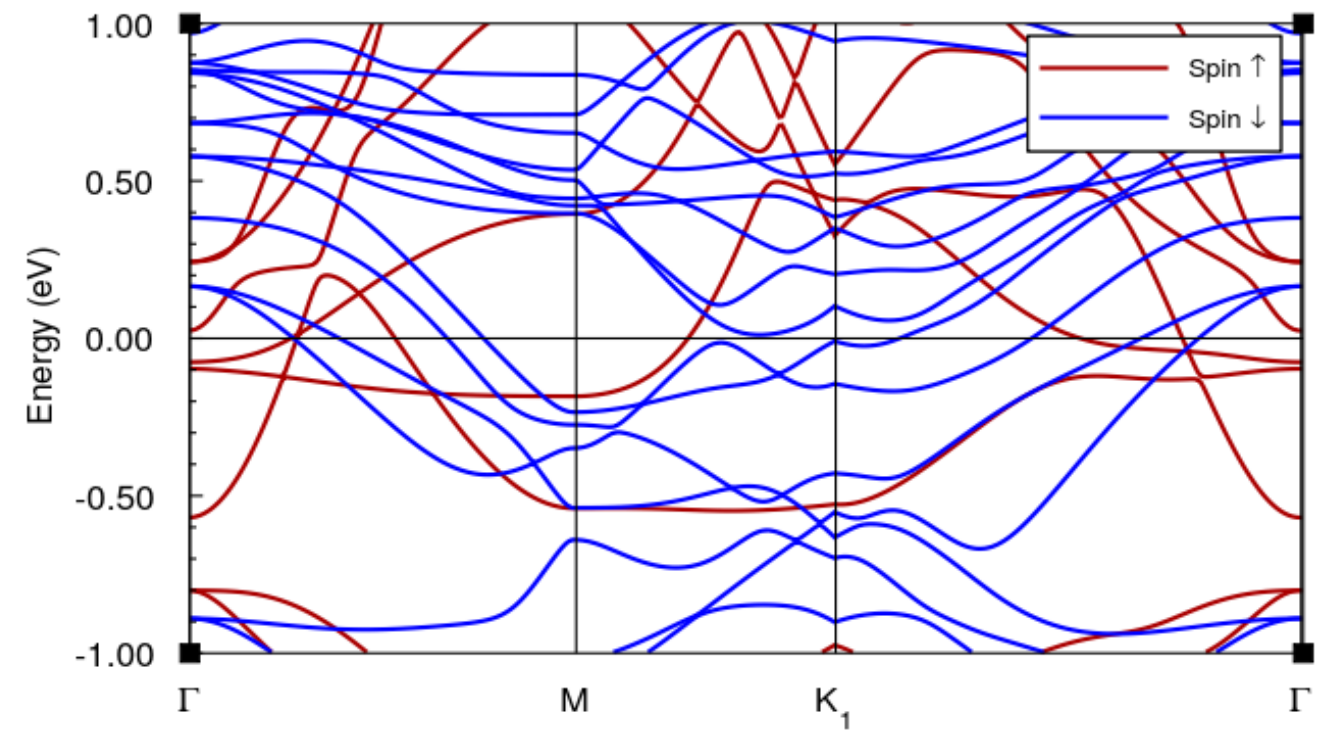

(c)

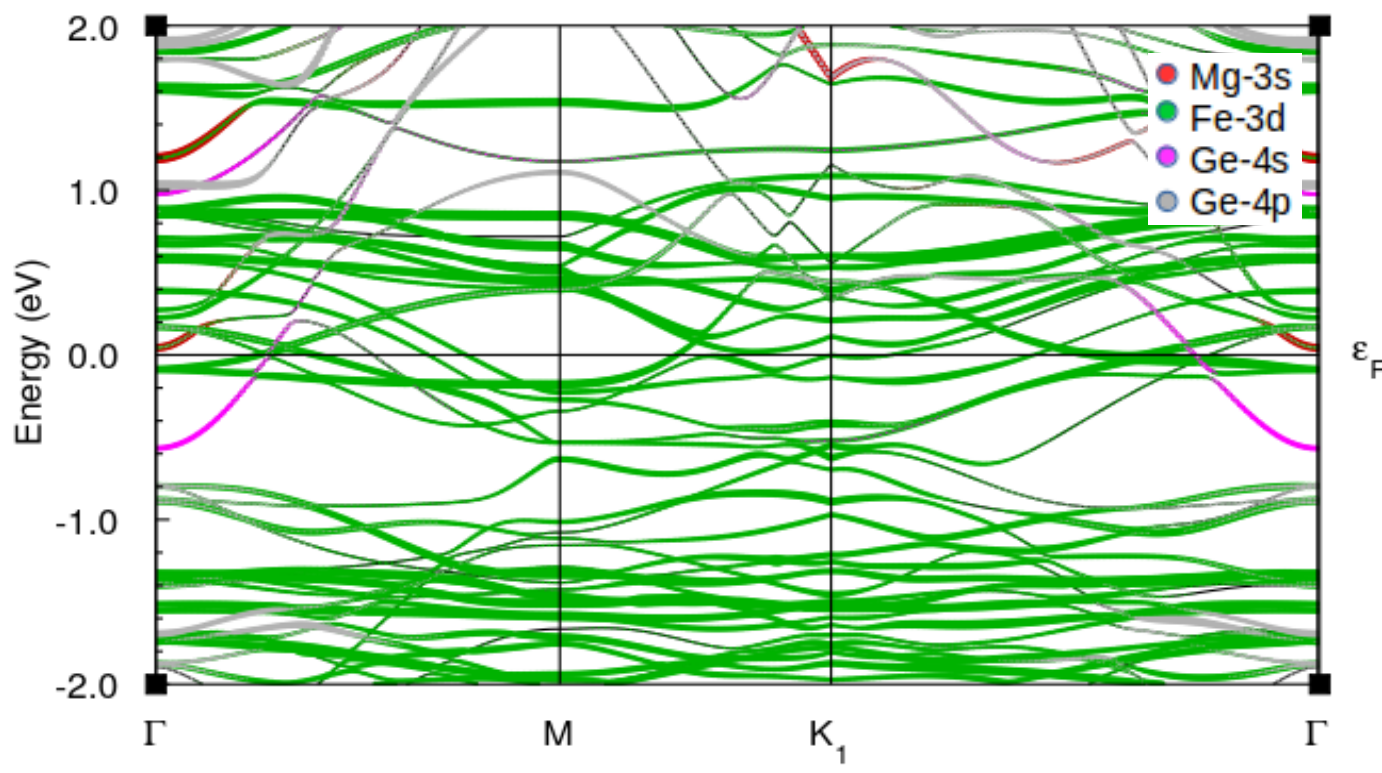

(b)

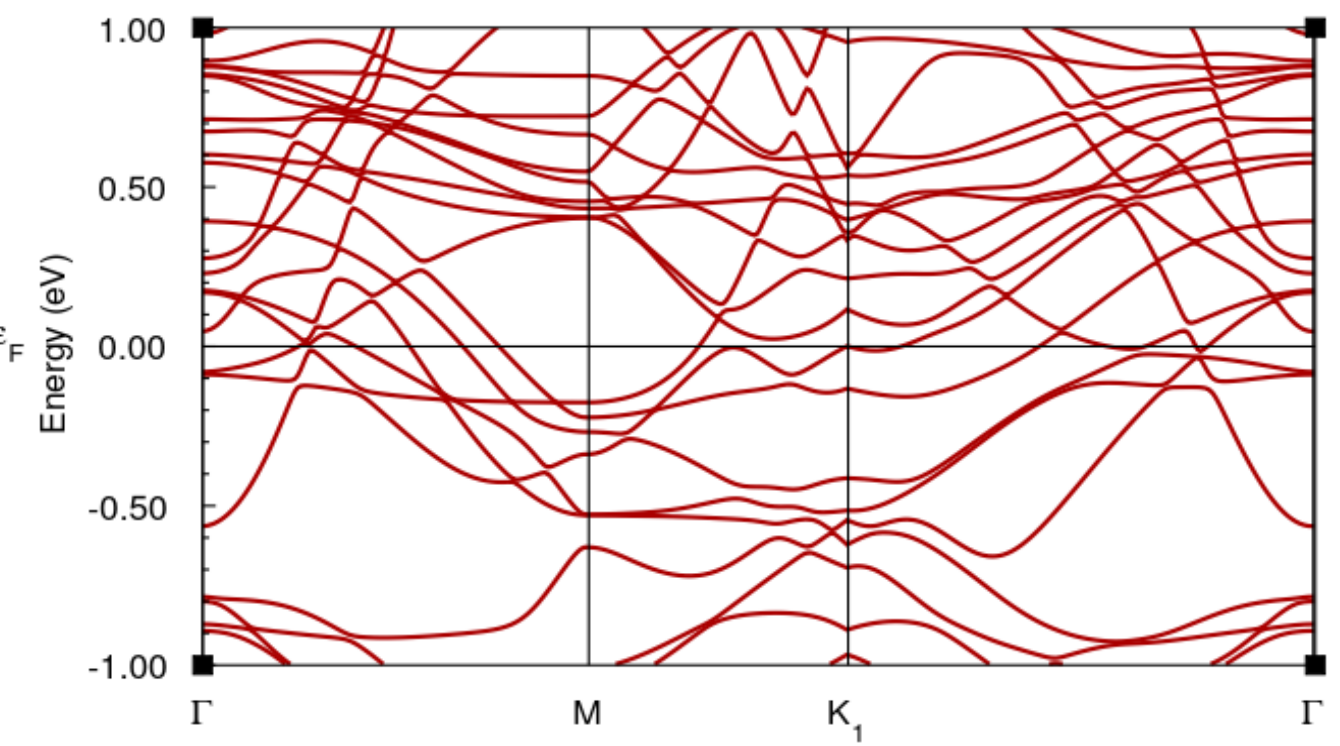

(d)

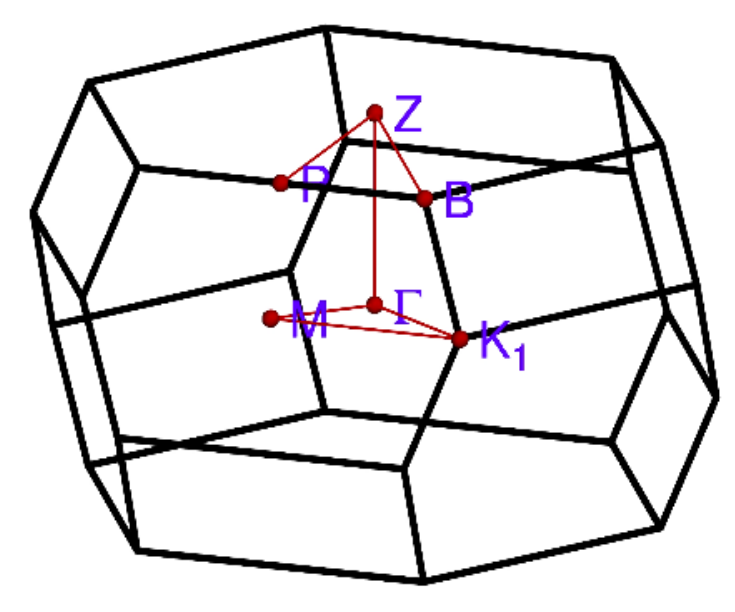

Crystals 2020

Fig 7: Band Structure (a) Scalar relativistics, (b) Full relativistics (c) Contribution of Mg-3s, Fe-3d, Ge-4p, Ge-4s (d) Brillouin zone 


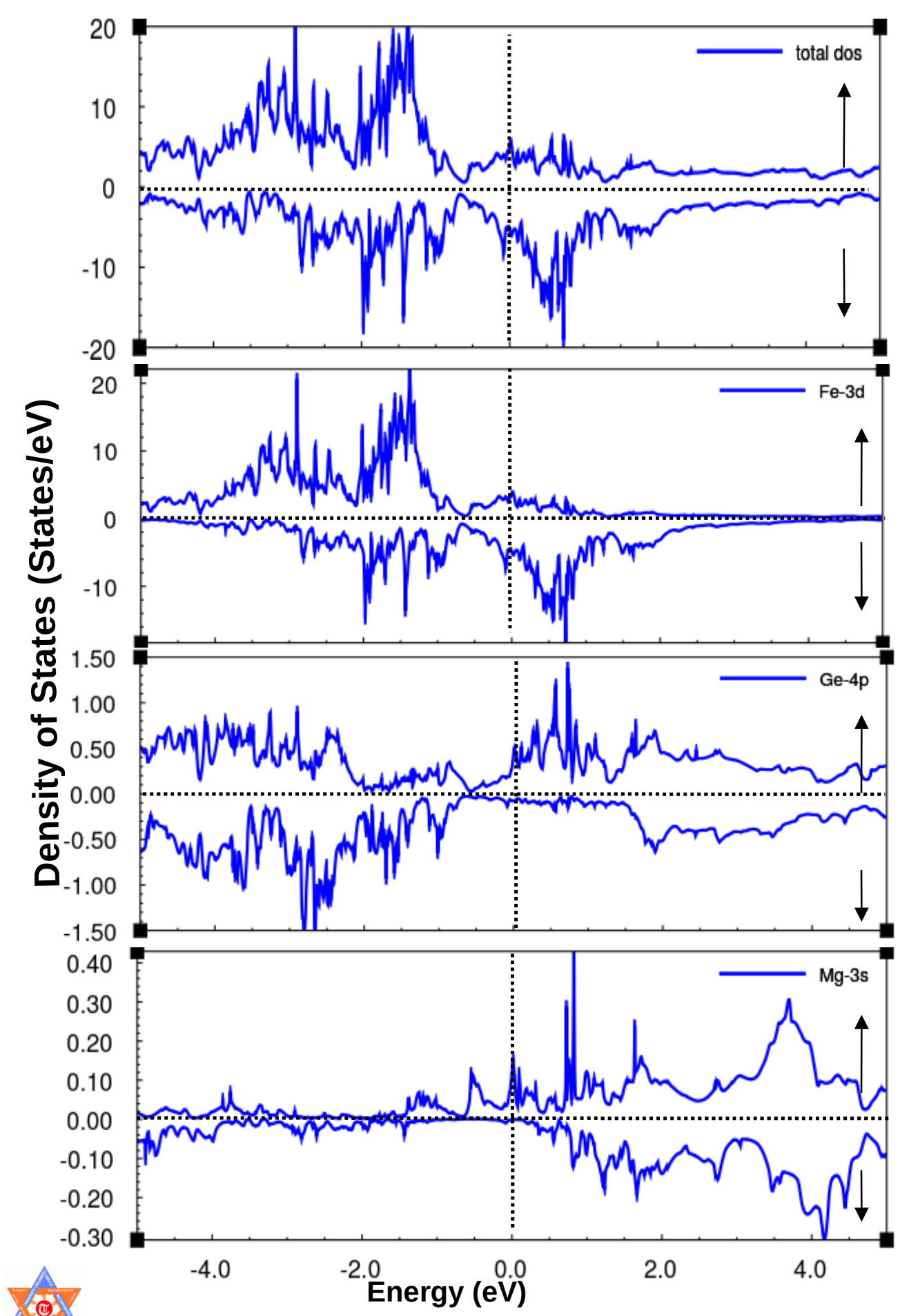

Fig 7: Density of States

$\square$ Remarks

$\rightarrow$ Fe-3d bands contribute mostly in the Fermi level.

$\rightarrow$ Hybridization between Fe-3d and Ge$4 p$ is observed at and around Fermi level.

$\rightarrow \mathrm{MgFe}_{6} \mathrm{Ge}_{4}$ is ferromagnetic material.

From DFT calculations magnetic moment per unit cell

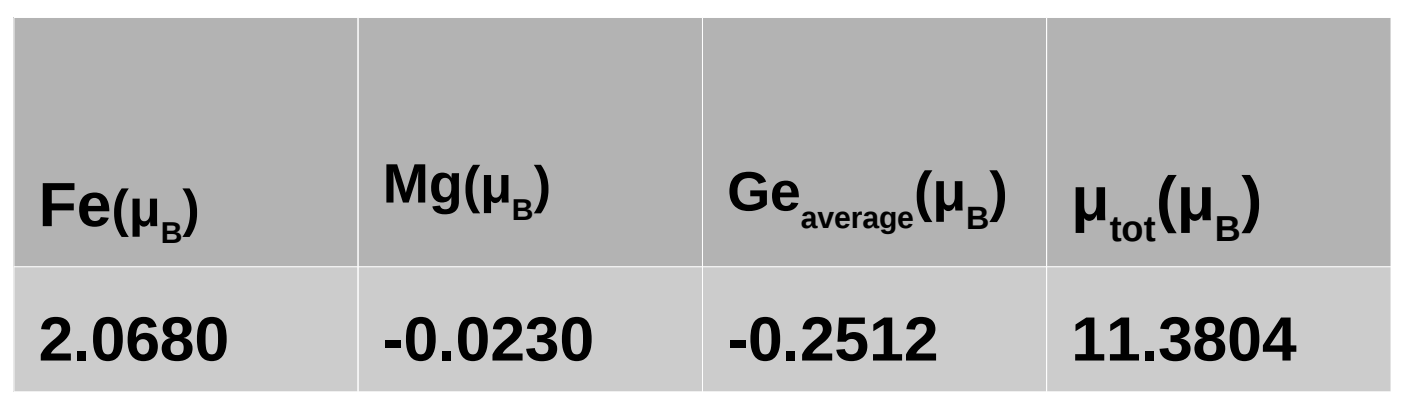

Crystals 2020 
Wannier Fitting

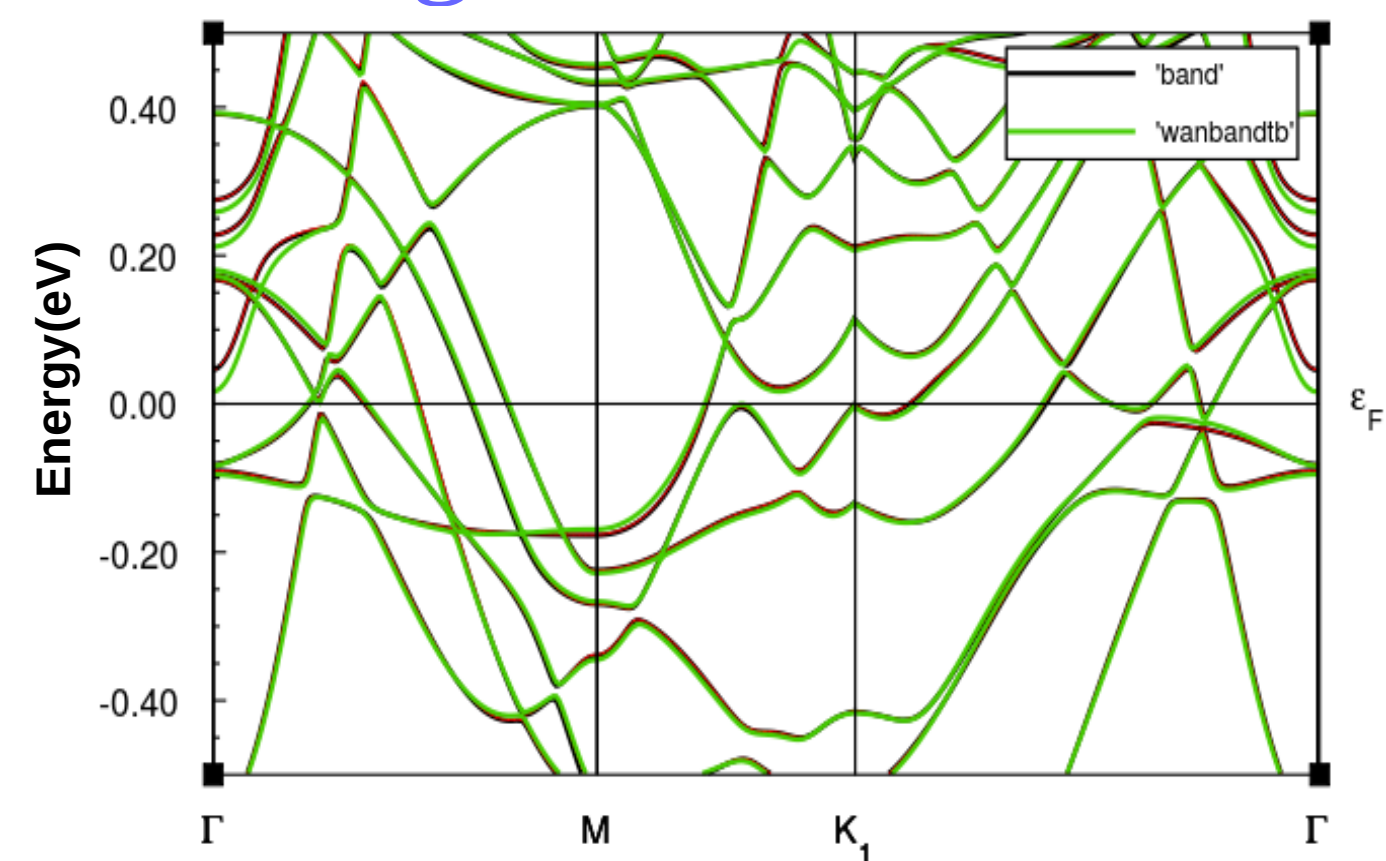

Fig 9: Wannier band mapping

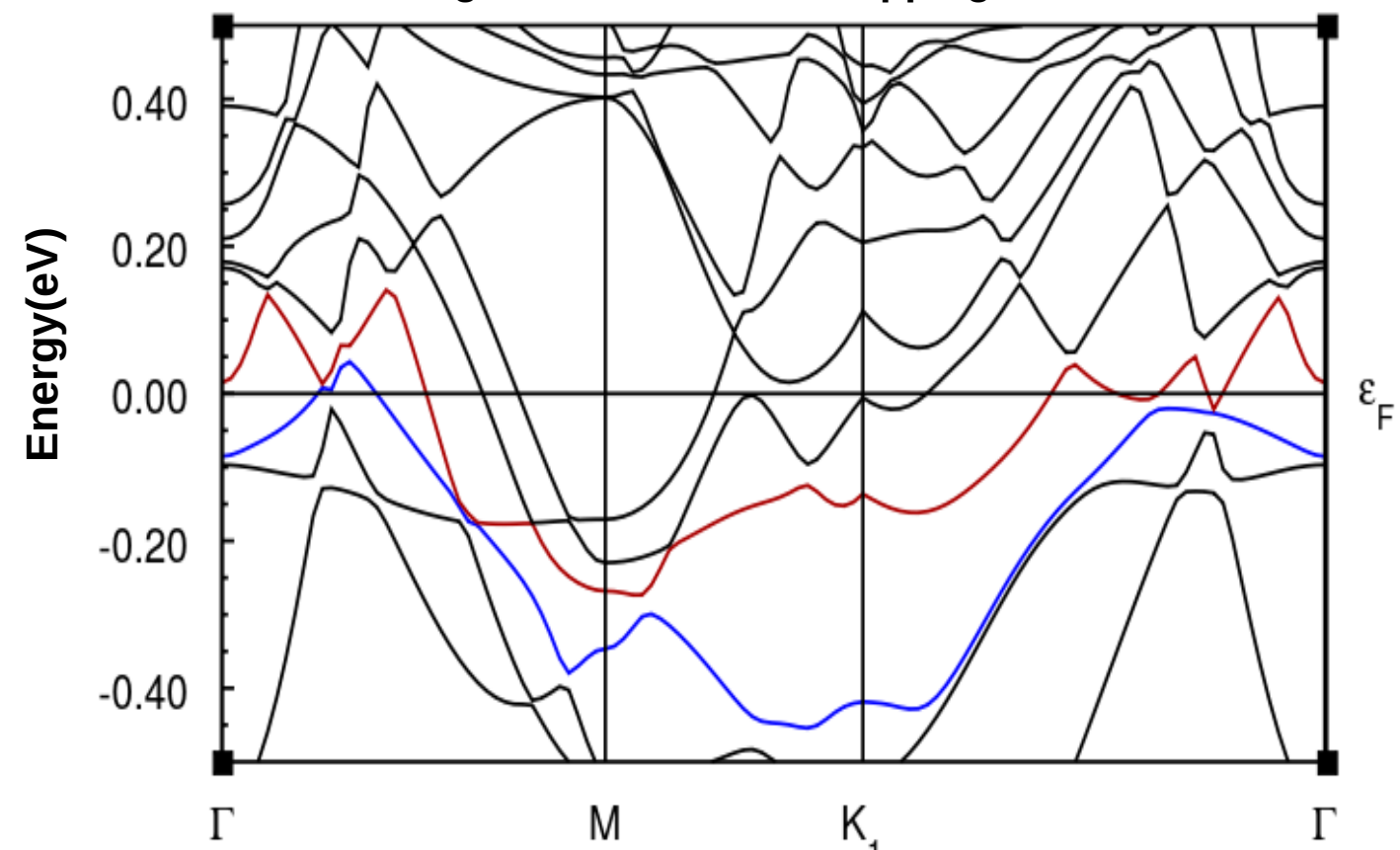

Fig 10: red band - CBM, blue band - VBM

Crystals 2020 


\section{Conclusions}

- $\mathrm{MgFe}_{6} \mathrm{Ge}_{4}$ is a ferromagnetic material found to be consistent with experiment.

Matar et al., Solid State Sciences, (2015)

- Study of DOS and band structure shows $\mathrm{MgFe}_{6} \mathrm{Ge}_{4}$ is metallic compound.

- Weyl crossing at $\Gamma$ and $M$ indicates Weyl semimettalic feature 


\section{References}

$\rightarrow$ L. Ye et al., Nature Phy., 25987, (2018)

$\rightarrow$ Kagome, Physics today, (2003)

$\rightarrow$ K. Koepernik and H. Eschrig, Phy. Rev. B 59, 1743, (1999)

$\rightarrow$ S. F. Matar et al., Solid State Sciences, 39, 82, (2015) 


\section{Acknowledgments}

- Prof. Dr. Binil Aryal, Head, Central Department of Physics, TU, Kirtipur, Nepal

- IFW-Dresden

- Kageshwori Manohara Municipality, Kathmandu, Nepal for the research grant

- Family and Friends
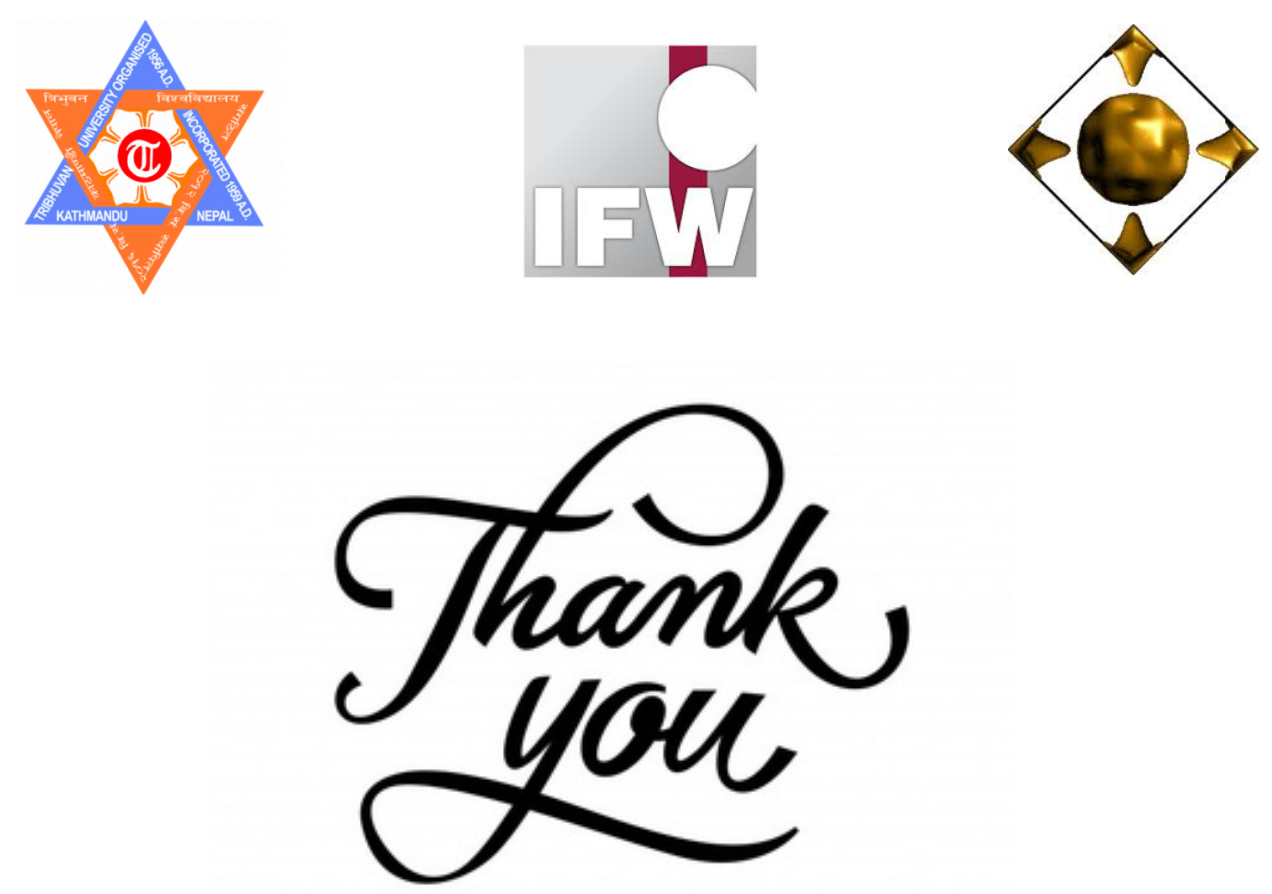